\title{
LA DOCENCIA UNIVERSITARIA: ESPECIFICIDAD, COMPLEJIDAD Y ALCANCES
}

J. Sánchez - Parga'

\section{Antecedentes y presupuestos}

$\mathrm{L}$

a formación universitaria no puede entenderse ni justificarse, en cuanto educación superior, sino como resultado de un proceso educativo, que presupone una educación primariay una educación secundaria, con sus propias especificidades educativas, y que la docencia universitaria completa y culmina. De manera general, como no se suele pensar la docencia universitaria a partir de sus condiciones de posibilidad, es decir desde la educación primaria y secundaria, puede ser ilustrativo para la argumentación que planteamos, repensar de manera breve y esquemática, las especificidades de la educación primaria y secundaria a partir de la perspectiva y retrospectiva de la educación universitaria.

Antes de entender el carácter superior de la docencia universitaria es preciso comprender el proceso de formación en el que se inscribe, y qué modelos de docencia presupone, ya que sin aquellos, o con una educación primaria y secundaria deficientes, la docencia universitaria sería o inútil o muy limitada en su eficiencia y formación. Ya que partimos del supuesto que la docencia universitaria no se caracteriza tanto y tan solo por los contenidos y formas de la "enseñanza" o contenido de los conocimientos, cuanto también por el nivel de desarrollo intelectual de los estudiantes, el cual solo puede ser resultado de una completa y coherente educación primaria y secundaria.

En otras palabras, quien ha recibido una deficiente educación secundaria y primaria, por muy buenas que sean las disposiciones e intereses y por muy buena que sea la docencia universitaria, esta nunca logrará cumplir sus objetivos académicos. De la misma manera que sin un previo desarrollo de la educación prima-

1 Investigador del CAAP, Asesor de la Dirección Académica de la Universidad Politécnica Salesiana Sede Quito. 
ria, sin un cumplimiento por parte de esta de sus específicos objetivos educativos y de aprendizaje, tampoco la educación secundaria podría lograr los suyos, puesto que presupone aquellos.

El objetivo de la educación primaria no es propiamente transmitir conocimientos sino más bien desarrollar las capacidades intelectuales del niño, referidas a aquellas categorías del pensamiento que son la condición de posibilidad, para que ulteriormente el niño pueda organizar los conocimientos correspondientes a dichas categorías mentales. Si el niño no ha previamente desarrollado las formas gramaticales de la lengua, en base a un amplio y regular uso de ellas, no podrá adquirir, usar ni poner en práctica las formas lógicas del pensamiento. Podrá llegar a saber lo que es la causalidad o una hipótesis, pero no podrá ejercer un pensamiento causal e hipotético. Ya que las formas lógicas se fundan sobre las gramaticales.

De la misma manera, sin un previo desarrollo, durante la educación primaria, de las categorías temporales (antes y después, lo nuevo y viejo, o antiguo, las duraciones largas, medias y cortas, las sucesiones, las simultaneidades, los cambios y sus diferentes ritmos, las frecuencias y repeticiones...), el niño no podrá después aprender y organizar las temporalidades presentes, pasadas y futuras; en definitiva nunca será capaz de pensar conocimientos históricos; todo lo más podrá retenerlos en la memoria, pero nunca como contenidos de un pensamiento histórico; es decir pensar la particular "realidad del pasado".

Exactamente lo mismo ocurre con las categorías espaciales (arriba y abajo, delante y detrás, dentro y fuera, lejos y cerca, las dimensiones de las distancias, encima y debajo...), sin cuyo desarrollo tampoco es posible enseñar, aprender y organizar los conocimientos geográficos.

Según esto la educación primaria abarca cinco grandes áreas de competencias específicas:

a) lengua o formas gramaticales de la lógica,

b) cálculo aritmético o formas mentales matemáticas,

c) categorías o capacidades del pensamiento espacial,

d) categorías o capacidades del pensamiento temporal,

e) categorías socio - relacionales (mundo, grupo, el otro, consigo mismo). 
Estas competencias específicas se desarrollarán y complementarán simultáneamente con las Ilamadas competencias de integración: oír / hablar, leer / escribir.

El dominio de ambos géneros de competencias deberá completarse al cabo de todo el ciclo de la educación primaria; siendo el nivel de dominio en el manejo de estas competencias lo que califica la educación primaria y su nivel de preparación para la secundaria.

Solo cumplidos los objetivos de la educación primaria podrá emprenderse una educación secundaria, cuya docencia se orienta no solo y no tanto a la transmisión cuanto a una enseñanza de conocimientos, cuyo aprendizaje consista en la organización de dichos conocimientos. El estudiante solo aprende los conocimiento que le enseñan en la medida que los organiza y relaciona de acuerdo a modalidades específicas. Solo esta específica organización de los conocimientos de la historia, la geografía, el lenguaje o la literatura, etc., al mismo tiempo que ayuda al estudiante a aprenderlos, en la medida que los organiza y comprende se desarrolla en el un pensamiento histórico, geográfico, matemático, literario o lingüístico...

Según esto la docencia en la educación secundaria no habrá de limitarse a enseñar conocimientos sino a enseñar cómo los conocimientos se organizan. Una cosa es enseñar los ríos de un país, sus fuentes, su curso, los cambios en sus cauces, sus afluentes y desembocadura, otra cosa muy diferente es enseñar el sistema fluvial de un país, y otra también muy diferente es enseñar dicho sistema fluvial, en relación con la orografía, el sistema montañoso, los sistemas climáticos y ecológicos, etc. Lo mismo se puede decir de la historia: una es la enseñanza de la Edad Media con todas sus características (modelo feudal, asentamiento rural, expansión del cristianismo...) y otra la enseñanza de la Edad Media como resultado de la transformación de la Edad Antigua (caída del imperio romano y dominación gótica) y preparación de la Edad Moderna; una cosa es "contar" la Revolución Francesa, con sus diferentes personajes y episodios, y otra muy distinta explicar los procesos de cambio que dieron lugar a la Revolución Francesa, todos los factores que confluyen en ella, y las transformaciones que ella misma provocara. 
De igual manera hay que tratar todas las "materias" o campos del saber de la educación secundaria. Y siempre recurriendo a una implementación de las competencias de integración desarrolladas en base a ejercicios de búsqueda de información, de organización o clasificación de informaciones (registrar, depurar y elaborar datos, registros de observación...): competencias funcionales y estructurantes. Se trata de seguir ejerciendo de manera más elaborada las funciones instrumentales de OíR, HABLAR, LEER Y ESCRIBIR, pero aplicada a la fase propia de la educación secundaria: organizar los conocimientos

Si al leer una novela, como por ejemplo, Pantaleón y las visitadoras de Vargas Llosa, no se enseña y orienta una lectura que permita, en primer lugar, situar el autor en el boom literario latinoamericano, comprender el autor y su época, en el escenario histórico de su propio país, y dicha novela en el contexto general de toda su obra; y en segundo lugar, registrar la acción principal, y las secundarias del relato; los protagonistas y personajes secundarios, sus distintas personalidades o caracteres, los escenarios, las descripciones, las ideas o valores transmitidos por el autor, los diferentes recursos literarios o estilísticos (metáforas, comparaciones, adjetivaciones, imágenes...) solo con una práctica habitual de este género de lectura podrá el estudiante desarrollar competencias de crítica literaria. Lo que después le servirá para analizar e interpretar otro género de textos.

No hay mejor "nivelación" de las diferentes formas de inteligencia que una buena educación primaria y secundaria, la cual no solo prepara sino que además condiciona la educación universitaria. Si la educación primaria y secundaria no han cumplido sus objetivos específicos en la formación del estudiante, la educación universitaria, por muy buena que sea, tendrá muy deficientes y limitados resultados.

En términos realistas, habrá que considerar que cada nivel superior de educación deberá suplir las posibles deficiencias del nivel inferior, para poder desempeñar sus específicas competencias educativas y lograr sus específicas efectividades y objetivos. En tal sentido la educación secundaria, sin declinar sus propias especificidades docentes, deberá completar las posibles limitaciones de una educación primaria, con la finalidad de lograr aquellas; 
así como la formación universitaria, sin abandonar tampoco sus propias especificidades y objetivos docentes y de formación superior, tendrá que completar eventuales defectos y suplir determinadas carencias de la educación secundaria, si quiere lograr sus propios objetivos.

Sin embargo, los defectos y limitaciones de la formación en los niveles educativos anteriores pueden ser de tal índole, y tal el grado de sus carencias, que la docencia superior (secundaria respecto de la primaria, y la universitaria respecto de la secundaria) no solo no podrá completar ni compensar, sino que tales deficiencias impedirán de manera absoluta que dicha educación superior logre sus más elementales efectos y rendimientos de su específica formación. En otras palabras, es totalmente inútil ya que los estudiantes carecen de las condiciones necesarias y requeridas para tal formación. Y lo peor que puede ocurrir es que tal formación universitaria implementada en estudiantes que no están capacitados para ella, no solo no logrará ser una real formación universitaria, degenerando en una suerte de educación secundaria en la universidad, sino que tampoco logrará complementar o completar las deficiencias de la educación secundaria.

\section{La docencia en la educación superior}

El objeto de la docencia universitaria no consiste en la exposición de los conocimientos de una ciencia, sino en cómo tal ciencia produce sus conocimientos, cuáles son los medios conceptuales y metodológicos para producir tales conocimientos, por qué se producen dichos conocimientos en ruptura, en confrontación o desarrollo respecto de otros conocimientos. Más que exponer el pensamiento de Maquiavelo, los conocimientos del marxismo, la teoría sociológica de Weber o las ideas antropológicas de Levi - Strauss, la docencia universitaria debe enseñar por qué, cómo, en qué circunstancias históricas e intelectuales o científicas se produjeron tales conocimientos; qué problemas se planteaban tales pensadores y de qué modo los resolvieron. En otras palabras la docencia universitaria más que enseñar los conocimientos de una ciencia enseña a pensar una determinada ciencia y a pensar científicamente sus conocimientos. 
Este objeto de la docencia universitaria lejos de limitarse a enseñar el modo de producción de una ciencia y sus conocimientos, debe adoptar la disposición científica de explicar el modo de producción de cada uno de sus conocimientos y conceptos, ya que los conocimientos e ideas ni nacen por generación espontánea, ni se transforman al azar, ni desaparecen o se pierden por sí solos. Explicar la producción, la genética o biografía, de los conocimientos es no tanto ni propiamente una enseñanza y aprendizaje científicos, cuanto una real práctica científica, donde los conocimientos son explicados y comprendidos.

Si cada vez resulta más necesario y también más urgente definir y reforzar la especificidad de la docencia universitaria, es porque las ciencias además de su normal acumulación de conocimientos, amplían cada vez más sus campos, se vuelven cada vez más complejas y diversificadas. Este proceso científico hace más difícil que la docencia universitaria pueda abarcar la ampliación de los conocimientos de cada ciencia; sin embargo, la formación universitaria debe garantizar una formación científica, y la preparación de profesionales, que si bien no abarcan todo el universo de conocimientos de una ciencia, sí son capaces de pensar científicamente. La sociología, la política, la psicología o el psicoanálisis, la biología o la física se han convertido en ciencias muy vastas, y muy difíciles de abarcar durante los períodos académicos de la Universidad. Por eso la docencia universitaria habrá de precisar cada vez más su principal objetivo: formar sociólogos, biólogos, antropólogos, físicos... que si bien no han aprendido todos los conocimientos de sus respectivas ciencias son muy capaces de pensar sociológicamente, antropológicamente, comunicacionalmente, psicoanalíticamente, políticamente, biológicamente..., la realidad. La docencia universitaria no consiste en enseñar los conocimientos de una ciencia sino en explicarlos, y en la medida que dichos conocimientos son comprendidos por el estudiante, de la docencia universitaria puede decirse que "enseña a pensarlos"; así como también sí quien comprende tales conocimientos puede aplicar sus resultados.

Esta especificidad de la docencia universitaria resulta tanto más pertinente por el hecho de que en la actualidad la posibilidad y disponibilidad de acceder a los conocimientos e informaciones sobre cualquier ciencia son enormes (libros, revistas, publicacio- 
nes de todo género, eventos diversos, informaciones y datos extraordinariamente elaborados, bases electrónicas de datos...); pero cada vez resulta en cambio más difícil el desarrollo científico y del pensamiento al interior de cada ciencia. Se puede llegar a aprender muchos conocimientos pero sin llegar a comprenderlos, ni saber pensarlos.

La docencia universitaria plantea un problema del orden de la "epistemología de la ciencia" con implicaciones académicas, y que podría formularse en los siguientes términos: si la ciencia, en lugar de un conjunto más o menos sistematizado de conocimientos relativos a un determinado campo del saber, es ante todo, y sobre todo, un proceso y modo particulares de producción de dichos conocimientos, la cuestión que se plantea es cómo "disciplinar" académicamente dicha ciencia, privilegiando la exposición, programación y docencia ya sea o bien del proceso y modo de producción de los conocimientos, o bien del sistema de todos los conocimientos de dicha ciencia, cómo se organizan, estructuran y relacionan entre sí.

De hecho, el orden teórico de una ciencia, los procesos y procedimientos, modos y medios en que produce y desarrolla sus conocimientos, no es el mismo que el orden de exposición de dichos conocimientos. Aquel puede relevar más bien de una genética de los conocimientos, mientras que este correspondería sobre todo a una sistemática más estructural de los conocimientos.

Este planteamiento al nivel general de una ciencia puede reproducirse al nivel de un determinado conocimiento, concepto, teoría particular o campo específico dentro de una misma ciencia: también en estos niveles más precisos se puede privilegiar un enfoque teórico-genético (cómo y por qué se produce un concepto, de qué manera se desarrolla teóricamente) o un enfoque expositivo - sistemático (el sentido y significación de dicho concepto en relación con los demás conceptos de un mismo marco o campo conceptual). En cualquier caso una docencia que tiene en cuenta ambos enfoques no se limita a enseñar conocimientos sino a pensarlos.

Estos dos órdenes, el teórico - genético y el sistemático estructura/ no son diferentes, sino más bien tan inseparables como complementarios del mismo proceso y conocimiento científi- 
cos, ya que en toda ciencia el modo de producción de los conocimientos inevitablemente comporta un progresivo grado de sistematización de dichos conocimientos. Sin embargo, ya la docencia universitaria, al disciplinar académicamente una ciencia por medio de su "pensum" o "curriculum" (la sociología, la biología o antropología), al traducirla en programas académicos, tiende más bien a privilegiar el orden de la exposición sistemática, de la estructuración y organización de los conocimientos por campos y áreas temáticos, pero tal procedimiento académico y de "disciplina de la ciencia" tiende a descuidar el orden teórico - genético de la ciencia en cuanto proceso y modo de producción de sus conocimientos.

Que las ciencias tengan que ser académicamente "disciplinadas", sistematizadas en "disciplinas", en campo particulares de conocimiento o materias al interior de cada ciencia, nada de esto implica que dicha ciencia haya de perder en la docencia universitaria su valor científico, que sus conocimientos se limiten a ser expuestos y transmitidos, enseñados y aprendidos, pero no pensados, porque no han sido realmente explicados y comprendidos.

Una nueva corriente metodológica en la docencia universitaria pretende o propone cambiar las materias o "asignaturas" por una enseñanza / aprendizaje "por problemas". Si este cambio se orienta a recuperar teóricamente la ciencia en cuanto proceso de producción de los conocimientos, que siempre es un proceso de producción de problemas y soluciones, tal "innovación" estaría respondiendo a las más correctas exigencias epistemológicas, tendientes a enseñar y aprender a pensar una ciencia y sus conocimientos, puesto que en una ciencia los conocimientos siempre han sido pensados como problemas y sus soluciones. Pero si, por el contrario, esta supuestamente nueva "docencia por problemas" se limita a enseñar tales problemas como se enseñaban los conocimientos, no en cuanto producidos sino en cuanto "datos", si tales problemas se enseñan aislados de los "campos de conocimiento" donde fueron producidos, eliminando su carácter sistemático y relación con otros problemas, en tal caso más que mejorar se estaría degradando aún más la docencia universitaria. Y en este sentido la docencia universitaria quedaría reducida a simples manuales o divulgaciones de las ciencias. 
Tomemos un primer ejemplo del psicoanálisis. Se puede "enseñar" el concepto de "asociaciones libres" como el método, por el cual es posible interpretar las elaboraciones del inconsciente durante la práctica psicoanalítica, ya que es a partir de tales "asociaciones libres" que el analizador, hablando de lo que se le ocurra, va dejándose hablar por su inconsciente, lo que permite al analista identificar e interpretar los significantes del discurso (no censurados por la conciencia) al margen de sus significados (que son elaboraciones conscientes). Pero también se puede enseñar cómo Freud produce el concepto de "asociaciones libres" a partir de las "censuras" o "resistencias inconscientes" de los analizados para hablar de sus reales problemas, y cómo será después a partir del discurso espontáneo del analizador, liberado de las resistencias conscientes, que por asociaciones libres, cuyos significantes aparecen liberados de la represión de la conciencia, se construye inconscientemente el discurso del analizador y el analista desconstruye e interpreta sus significantes.

Otro ejemplo, tomado de la sociología actual es el concepto de "movimientos sociales". A partir de su definición como "la constitución de actores sociales en sus específicas prácticas y discursos", se pueden explicar los distintos significados de cada uno de los componentes conceptuales y correspondientes relaciones conceptuales de dicha definición. Pero este mismo concepto puede ser enseñado a partir de la transformación del paradigma estructuralista, que explica los procesos sociales y cómo los diferentes sectores de la sociedad se encuentran sujetos a ellos, en un paradigma post-estructuralista ("el regreso del actor"), donde el análisis se desplaza de las estructuras y los procesos sociales a las actuaciones en ellos de los diferentes grupos, clases y sectores de la sociedad, tomando como referente analítico tales actuaciones, prácticas y discursos, por los cuales dichos actores se constituyen e identifican, se relacionan, movilizan y luchan entre sí.

Se trata de dos modos de "enseñar" la ciencia que lejos de diferentes son complementarios; una docencia que no se limite a enseñar conocimientos sino que los explica para pensarlos, ya que razona y argumenta cómo fueron producidos, por qué y para qué. Sin embargo, tres razones recomendarían enfatizar el enfoque 
teórico-genético de producción de conocimientos sin detrimento del enfoque más sistemático:

a) Permitiría corregir lo que parece haber sido la tendencia más generalizada o dominante de la docencia universitaria: enseñar conocimientos de las distintas ciencias y no tanto explicar el modo, el proceso y los procedimientos de producción de dichos conocimientos.

b) Si la especificidad de la formación universitaria es la formación científica, basada esta en el desarrollo del pensamiento científico y no tanto en la ampliación de conocimientos científicos, sería necesario priorizar el enfoque teórico sobre el sistemático. Ya que es aquel pensamiento el que realmente forma científicamente y prepara para la investigación.

c) Dados los actuales desarrollos de las ciencias, la ampliación, diversificación y complejidad de sus campos de conocimientos, cada vez resulta más difícil que durante el período de una carrera se puedan abarcar tantos y tan especializados conocimientos de una misma ciencia. Siendo por ello necesario implementar una "economía académica" de cada ciencia no es menos necesario que el estudiante y futuro profesional, aunque no posea todos los actuales conocimientos de dicha ciencia, tenga la suficiente formación científica.

Una buena docencia universitaria y formación científica del estudiante deberían garantizar e integrar la doble comprensión del proceso científico y de los "usos de la ciencia".

\section{Docencia o pedagogía}

Si el objeto de la docencia universitaria es siempre una ciencia, "enseñar a pensar" dicha ciencia y pensar sus conocimientos, que tal ciencia produce y el modo cómo los produce, será la misma ciencia la que determina y precisa las formas y procedimientos de su explicación y comprensión. Cada ciencia, por consiguiente, posee su propia "metodología" (no pedagogía) y modalidades de explicación de acuerdo a la particular generación / desarrollo y organización de sus conocimientos. Cualquier pedagogía o didáctica 
ajena a una ciencia no solo será incapaz de explicar dicha ciencia, puesto que una ciencia únicamente puede ser pensada desde sí misma, sino que cualquier intento de pedagogización o de instrumentación didáctica de los conocimientos de una ciencia se limitarían a meras recetas educativas. Lo que algunos denominan "enseñar a pensar" una ciencia no es otra cosa, en realidad, que "explicar una ciencia", para que en lugar de ser "aprendida" sea "comprendida".

Ya que lo aprendido puede olvidarse, mientras que lo comprendido forma ya parte del pensamiento, y esta "formación del pensamiento" no es objeto de olvido.

Los intentos de pedagogización de la docencia universitaria son por lo general resultados de una pérdida y precarización del pensamiento científico propio de cada ciencia. Cuando las ciencias se empobrecen teóricamente, cuando sus sistemas conceptuales se vuelven nocionales, simples ideas e informaciones para designar la realidad pero no para comprenderla y explicarla y producir nuevos conocimientos sobre ella, tal penuria epistemológica lleva a recurrir a la pedagogía, como si una ciencia o técnica subsidiaria pudiera compensar las deficiencias de la práctica y de los usos de otras ciencias.

Consecuentemente, siendo el reto y responsabilidad de la docencia universitaria no tanto enseñar los conocimientos de una ciencia sino "enseñar a pensar", es decir explicar, dicha ciencia, la formación del estudiante universitario no es aprender los conocimientos de una ciencia, para lo cual pudiera servirle una pedagogía, sino aprender a pensar dichos conocimientos desde dicha ciencia; es decir comprenderla.

Así planteado el problema de la docencia universitaria no es tanto de orden pedagógico cuanto científico. Solo el docente que conoce su ciencia, que sabe cómo se producen los conocimientos en ella, y cómo se organizan, explicándose todos ellos entre sí, es capaz de implementar una competente y eficaz docencia universitaria. No hay una pedagogía capaz de enseñar a pensar una ciencia; solo desde cada ciencia particular se puede enseñar y aprender a pensar científicamente.

Incluso dentro de las ciencias sociales una es la epistemología de la sociología, otra la epistemología del psicoanálisis, otras 
diferentes la de la lingüística o la antropología, ya que diferentes fueron los saberes, que permitieron producir sus diferentes objetos teóricos (el inconsciente, la diferencia, la distinción entre lengua y lenguaje...). Son estos saberes que los estudiantes habrán de adquirir al mismo tiempo que comprenden los conocimientos de la respectiva ciencia.

El modo cómo la sociología produce y organiza sus conocimientos no es el mismo del modo de producir y organizar los conocimientos de la antropología, el psicoanálisis o la lingüística. Si el estudiante no comprende estos modos de producción y organización de los conocimientos de la ciencia, podrá aprender en el mejor de los casos muchos conocimientos de sociología, de antropología, de psicoanálisis o lingüística, pero nunca llegará a pensar sociológicamente, antropológicamente, psicoanalíticamente la realidad y sus problemas.

El actual abuso, tan inflacionario como equívoco, de la noción de epistemología ha contribuido a olvidar que solo una ciencia, que ha producido y elaborado su propio objeto teórico, y a partir del cual ha desarrollado sus propios conceptos y conocimientos, solo a tal ciencia cabe atribuir una epistemología; es decir una forma particular de saber y pensar sus conocimientos, diferente de las formas de pensar y saber de otras ciencias.

Según esto, ninguna pedagogía podrá enseñar ni servir a un antropólogo, sociólogo o psicoanalista para explicar sus respectivas ciencias. A no ser que dicho pedagogo sea tan buen sociólogo, antropólogo o psicoanalista como pedagogo. Cualquiera, después de haber leído un libro de sociología, de antropología o de psicoanálisis, y de haberlo entendido, podría atreverse a enseñar los conocimientos que aprendió con tal lectura, pero no podrá enseñar a pensar dichos conocimientos, explicar cómo y por qué fueron producidos.

Sobre la pedagogía de una ciencia se requiere precisar que las ciencias tienen su propia "pedagogía", es decir, su específico modo de explicarse y ser comprendidas; por ello es importante reconocer, que el mismo pensamiento científico posee sus peculiares dificultades, y que no siempre el estudiante se encuentra suficientemente preparado por su formación anterior (deficiente educación primaria) para comprender los presupuestos epistemológi- 
cos, los componentes teóricos y conceptuales y niveles de abstracción y procedimientos lógicos de una ciencia. La docencia universitaria lejos de desconocer esta situación, probablemente más generalizada de lo que supone la mayor parte de los docentes, habrá de ser tenida muy en cuenta. Pero esto no significa que por procedimientos pedagógicos e instrumentos didácticos se facilite al estudiante la comprensión de la ciencia. Una docencia universitaria que facilita las dificultades de una ciencia para su mejor aprendizaje, lejos de enseñar una ciencia se limita a transmitir una versión divulgada de ella. Lo que entonces se aprende es una "sociología de quiosco", una "antropología de quiosco", un "psicoanálisis de quiosco", recetarios de nociones e informaciones y simples trucos apropiados a la vanalización de cada una de dichas ciencias.

El docente universitario no es ningún "facilitador" científico que vanaliza, descafeína y hace digestiva una ciencia para que pueda ser aprendida "sin esfuerzo"; como si su función docente se redujera a implementar un método tipo "aprenda sociología en 30 días", "aprenda el psicoanálisis sin esfuerzo". Todo lo contrario, su responsabilidad docente y hasta científica (más que pedagógica) consiste en que los estudiantes lleguen a comprender (más que aprender) las dificultades de la ciencia, o comprender una ciencia con todas sus dificultades, ya que cuanto más difíciles son las dificultades que comprenden de una ciencia tanto mejor se forman y mejor piensan científicamente.

De hecho, y cada vez con más frecuencia, nos encontramos con propuestas educativas y con trabajos académicos, que en nombre de una ciencia no hacen más que producir y traficar chatarra científica: ciencias vaciadas de pensamiento y conocimientos sin teoría. No es raro que aparezcan explicaciones del desarrollo capitalista, que ignoran "el capital" como objeto teórico, psicoanálisis sin inconsciente, sociologías que desconocen el "hecho social" o antropologías que nunca han pensado "la diferencia" en cuanto concepto teórico, ya que están convencidas que las diferencias entre sociedades y culturas se palpan y se ven, y por consiguiente no es necesario pensar estas desde aquella.

El gran reto no pedagógico de la docencia universitaria consiste específicamente en cómo explicar y lograr que el estudiante 
comprenda las mayores dificultades de cada ciencia. Esto, y no otra cosa, es lo que enseña a pensar, y en esto se define la específica formación universitaria. Enseñar a pensar no es más que "explicar y comprender".

Nada de ello impide, sin embargo, que la docencia universitaria recurra a procedimientos comunicacionales, que ayuden a la enseñanza / aprendizaje de una ciencia, sobre todo si se tienen en cuenta las deficiencias en la formación intelectual de los estudiantes. Defectos de un pensamiento no habituado a los análisis y las síntesis, a la organización y esquematización de los conocimientos, a los procedimientos lógicos y argumentativos, a la racionalización, la conceptualización y la abstracción, todo esto que es parte del conocimiento científico es parte también de sus presupuestos, y por consiguiente de un tratamiento metodológico. Pero no de la pedagogía. Que el docente proponga en cada clase un esquema de su curso, que establezca las conexiones y continuidades entre los sucesivos desarrollos temáticos y problemáticos, que amplíe las múltiples relaciones entre diferentes temas de su programa, entre unos conocimientos y otros, que en el trabajo de la bibliografía oriente y guíe la lectura con indicadores o cuestiones de referencia, que faciliten la comprensión de los textos; todos estos y otros muchos recursos serán siempre importantes y necesarios para que la docencia universitaria lejos de abdicar de su especificidad científica, apoye el aprendizaje y el desarrollo del pensamiento en los estudiantes.

Los desarrollos precedentes de ninguna manera impiden que la docencia universitaria pueda adoptar recursos y orientaciones o procedimientos pedagógicos sin detrimento de su específica condición y eficacia académicas. Más aún, teniendo en cuenta las posibles y hasta generalizadas deficiencias de la educación secundaria, dadas las limitaciones en la formación preuniversitaria, será hasta necesario que la docencia en la Universidad compense y complete los bajos niveles de dicha formación, no para suplirla o sustituirla, sino con la finalidad de lograr una mejor eficiencia docente. 


\section{Docencia e investigación universitarias}

Aunque nadie, en ningún medio académico, se atreva a cuestionar que la investigación sea un elemento tan fundamental como específico de la formación universitaria, no es fácil identificar el lugar académico de la investigación o las prácticas específicas de investigación en dicho proceso de la formación superior.

Esta dificultad se encuentra, a su vez, atravesada por otra relativa a las imprecisas o vaporosas ideas sobre lo que es la investigación. Para unos todo o cualquier cosa puede ser investigación, y hasta llaman investigar la elaboración de monografías o recopilación de información y datos sobre un determinado tema en la educación secundaria. Sin desconocer el valor de estos trabajos escolares o académicos, y su importancia para la preparación de las futuras prácticas de investigación en la Universidad, la investigación universitaria se entiende siempre en términos de una investigación científica, realizada en el campo de una ciencia particular, y de acuerdo a un determinado modo y metodología de producción de conocimientos. Se trata de prácticas de investigación en las que el mismo investigador se encuentra "sujeto" a un determinado proceso científico.

Es en este preciso sentido que nos parece importante mostrar la estrecha articulación entre la docencia universitaria, en cuanto enseñanza / aprendizaje científico, de los modos de producción de los conocimientos de una ciencia, y las prácticas investigativas en el marco de dicha ciencia. Lo que con ello se pretende demostrar es que solo una docencia implementada en los términos desarrollados, y con su especificidad académicamente universitaria, constituye la condición necesaria para la formación de investigadores. Por el contrario, una docencia que se limite a una enseñanza y aprendizaje de conocimientos lejos de preparar para la formación de investigadores, se constituye en un serio obstáculo para la realización de posibles investigaciones. Es toda la diferencia que existe entre manejar conocimientos en cuanto "datos" o "hechos" y conocimientos en cuanto "producidos".

Se suele atribuir una mayor o menor importancia y valoración académicas a las llamadas "memorias" o "tesis" de grado, y no sin razón, puesto que constituyen la mejor oportunidad para 
que el estudiante, contando con los necesarios apoyos y requerimientos académicos, pueda hacer la experiencia científica de producir conocimientos en el área de su ciencia y especialidad o carrera. Sin esta experiencia académica, con todas sus exigencias y apoyos docentes o recursos disponibles durante su formación, difícilmente podrá después el estudiante llevar a cabo una práctica científica de esta índole, lo que por otra parte supondrá siempre un vacío en su formación profesional.

No son tan frecuentes las ocasiones y los profesores que en sus respectivas materias, más allá de alguna composición o ensayo sobre temas particulares, implementan ejercicios de investigación con un cierto rigor científico y metodológico.

Lo que de manera generalizada se constata y llama la atención es la distancia, diferencia y separación entre la docencia y la investigación, en cuanto prácticas académicas no solo distintas sino también carentes de relación alguna entre ellas. Pero esto no significa que la docencia universitaria no tenga nada que ver con la investigación, a no ser en cuanto los presupuestos implícitos o determinadas condiciones indirectas para las posibles prácticas investigativas. $\mathrm{Si}$, en contra de lo que se acaba de sostener, la docencia no se ejerce como una explicación del proceso científico, si se limita a enseñar los conocimientos de una ciencia, y no a explicar cómo y por qué, con cuáles recursos conceptuales, en ruptura o en contradicción con qué otros conocimientos, cómo dicha ciencia ha ido evolucionando conceptual y teóricamente, sin una docencia así implementada, sin una ciencia así explicada y razonada, el estudiante podrá haber acumulado muchos conocimientos de dicha ciencia, pero desconociendo el modo de su producción, no estará capacitado para investigar.

Solo la docencia que razona cómo se producen los conocimientos, explica ya cómo investigar. $Y$ solo el estudiante que ha comprendido cómo se producen los conocimientos de la ciencia será capaz él mismo de producir conocimiento en ella; es decir, será capaz de investigar. De lo contrario, poco importa que el estudiante tenga muchos y buenos cursos sobre "métodos y técnicas" de investigación, o seminarios sobre la "elaboración " o el "diseño" de tesis. Todos estos recursos instrumentales de la investigación científica nunca compensarán la ignorancia del modo de producir 
los conocimientos de una determinada ciencia, aun cuando probablemente conozca tales conocimientos.

Este defecto de la docencia universitaria se manifiesta ya cuando los estudiantes, al tener que diseñar su plan de tesis, o al elaborar su proyecto de investigación, se enfrentan con el tratamiento del "marco teórico". Esta suerte de requisito metodológico, consecutivo a la definición del problema de la investigación o de la "construcción del objeto" de investigación, se convierte en una clase de protocolo de difícil tratamiento, un cuerpo extraño o ejercicio especulativo, que probablemente nada tiene que ver con la futura, real y concreta, realización y desarrollo de la investigación. La explicación de tal situación es obvia: los docentes "enseñan" la teoría de la ciencia, exponiendo y definiendo conceptos, y en el mejor de los casos mostrando cómo tales conceptos se relacionan, se explican e interpretan entre ellos; pero al no explicar el docente cómo todos esos conceptos han sido producidos y elaborados, qué razones y razonamientos han intervenido en su producción y elaboración, a partir de qué problemas y de qué soluciones, y cómo se han ido integrando sistemáticamente en una teoría, difícilmente el estudiante sabrá producir un problema, para investigar con él determinadas realidades o hechos.

El "marco teórico" no es más que ese sistema de conceptos que permiten definir, elaborar o construir un problema u objeto de investigación. Identificar ese mismo sistema de conceptos que como "instrumental teórico" intervendrá tanto en la producción como en la elaboración e interpretación de los datos, informaciones y conocimientos producidos en el curso de la investigación. En términos comparativos, el "marco teórico" no es otra cosa para el investigador que esa caja de herramientas indispensable para un fontanero o electricista.

Pero este uso investigativo de los conceptos ha presupuesto una docencia, que en lugar de limitarse a definir conceptos (cuando los conceptos lejos de "definirse" solo pueden comprenderse y explicarse al interior de un sistema de "indefinidas" relaciones conceptuales, con otros conceptos) los ha explicado en relación con otros conceptos, para comprender e interpretar las realidades a las que corresponden.

Obviamente no todas las áreas de una ciencia, ni todas los campos de conocimiento de dicha ciencia, no todas las "discipli- 
nas", "materias" o cursos se prestan a la misma orientación investigativa de su docencia. Es en las áreas más teóricas donde la docencia puede explicar las bases, los presupuestos y conceptos fundamentales del conocimiento de dicha ciencia pero también de sus posibilidades investigativas. Solo una buena formación teórica de la sociología explica al estudiante que un crimen, por ejemplo, no es un simple acto de violencia, un accidente, un suceso, una transgresión, sino un "hecho social" producido en y por una determinada sociedad, y cuya comprensión y explicaciones en última instancia hay que buscarlas en dicha sociedad. La docencia en cualquier área del conocimiento de una ciencia deberá ilustrar ampliamente todas las posibilidades de investigación que todavía plantea dicha área científica, los problemas aún no planteados y otros tampoco definitivamente resueltos. Si la investigación es la "agenda" de toda ciencia, las cuestiones pendientes resultado de los conocimientos recientes, la docencia habrá de ser implementada con estas perspectivas.

Según esto resulta claro que, en general, sean los docentes, que tienen experiencia más o menos amplia de investigadores, quienes con más competencia y eficacia pueden desempeñar una docencia universitaria. Quienes tienen experiencia de cómo se producen los conocimientos serían los mejor preparados - ¿los únicos?- capacitados para explicar no solo los conocimientos en cuanto producidos científicamente sino también su reproducción y desarrollo.

\section{Docencia y evaluación}

Entendida la docencia universitaria de acuerdo a los precedentes desarrollos, y considerada fundamentalmente no como una enseñanza / aprendizaje de conocimientos sino en cuanto explicación / comprensión de producción de conocimientos, cómo se producen y piensan los conocimientos de una ciencia, resulta obvio que la evaluación de tal docencia universitaria no puede limitarse a examinar los conocimientos aprendidos por el estudiante, sino el nivel y la forma, o más exactamente, la calidad de comprensión y de explicación, que el estudiante posee de los conocimientos, la comprensión y explicación de sus modos de producción. 
Este modelo de evaluación no solo se limita a examinar el desarrollo del pensamiento científico del estudiante, y en qué medida el estudiante, por ejemplo, de sociología o antropología, aprende a pensar sociológicamente o antropológicamente los conocimientos aprendidos, sino que dicho modelo de evaluación pone a prueba además el valor y calidad de la misma docencia. Así planteada la evaluación de la docencia en su doble eje de la enseñanza y el aprendizaje, será preciso planificarla con tiempo y modalidades particulares.

Tres razones justifican actualmente una nueva modalidad de evaluación de la docencia universitaria: a) la diversificación y complejidad de las ciencias y tecnologías; b) las exigencias tanto académicas como sociales de un mayor rendimiento del proceso de enseñanza - aprendizaje; c) y en general la necesidad de compensar una más limitada disponibilidad y dedicación del estudiante a sus desempeños académicos.

Convencionalmente se suelen separar el momento y procedimiento de evaluación del estudiante y del docente, y ambos del mismo proceso de explicación - comprensión. Sin excluir estas formas de evaluación (exámenes, pruebas, controles, formularios de evaluación docente - estudiantil), es importante definir un modelo complementario de evaluación de la docencia - aprendizaje, mucho más directa e inmediatamente vinculado a la acción - proceso de enseñanza - aprendizaje, que opere como seguimiento en la misma aula de dicho proceso, y que simultáneamente sirva de control tanto para el docente como para el estudiantes de sus respectivos desempeños.

Las pruebas o exámenes trimestrales o semestrales pueden resultar demasiado tardíos, y de consecuencias en parte ya irreparables, para evaluar un proceso de docencia aprendizaje, cuando ya muchos elementos o deficientes resultados de dicho proceso son irremediables.

De otra parte, si se considera que la docencia universitaria, más que transmitir conocimientos propios de una ciencia, más bien comparte el proceso de producción científica de tales conocimientos (cómo, por qué, en qué circunstancias tales conocimientos fueron producidos, con qué instrumentos conceptuales o recursos científicos, en ruptura y polémica con qué otras teorías...), en- 
tonces se vuelve más importante y necesario un modelo de evaluación, que en lugar de examinar qué conocimientos el estudiante conoce o recuerda, trata más bien de estimar cómo piensa tales conocimientos; qué niveles de comprensión y explicación posee de ellos.

En este sentido parece tan interesante como urgente un modelo y procedimiento de evaluación docente - estudiantil estrechamente vinculado al mismo proceso de explicación - comprensión, y que sea una suerte de seguimiento mutuo de dicho proceso, de "reflexión científica (más que de "reflexión pedagógica") sobre el "orden de comunicación" del pensamiento científico.

Según esto, un modelo de evaluación de la docencia universitaria pasa necesariamente por un desarrollo y perfeccionamiento de dicha "docencia académica", que no se limite a capacitar pedagógica o didácticamente a los docentes universitarios, sino a mejorar la comprensión de las relaciones entre el "orden de la exposición" y el "orden teórico" de cada ciencia. Sobre todo si se tiene en cuenta que la formación universitaria no consiste tanto en la cantidad de conocimientos de una ciencia cuanto en la capacidad de pensar científicamente dichos conocimientos.

Más que de una evaluación propiamente dicha se trata de un seguimiento investigativo muy directo y sostenido del mismo proceso de docencia / aprendizaje, que en la misma clase o aula, al cabo de cada unidad del programa, el docente pudiera hacer una estimación en profundidad, y en base a muestras aleatorias de los estudiantes, para evaluar el nivel de comprensión por parte del estudiantes de la explicación por parte del docente y del desarrollo de la materia tratada.

Tal estimación / evaluación puede realizarse en base de una serie de cuestiones claves, y a partir de las cuales se puede obtener la información suficiente o las respuestas sintomáticas del nivel de comprensión más que de aprendizaje propiamente dicho. Este procedimiento puede servir tanto de evaluación cuanto de auto-evaluación por parte del docente como por parte del mismo estudiante, pudiéndose identificar qué contenidos, aspectos o problemas fueron entendidos y qué nivel de comprensión, cuáles no fueron suficientemente expuestos o explicados o razonados. Este procedimiento de evaluación proporciona además una evaluación 
general o promedial del aprendizaje de todo el curso, pero también ofrece una evaluación mucho más particularizada de cada estudiante.

Este procedimiento evaluativo puede ser muy orientador de las pruebas y exámenes regulares, a los que el alumno estará sujeto, y en parte puede servir de preparación para ellos.

En algunas universidades los estudios de "agregación para la enseñanza superior" contienen siempre programas relativos a "los enfoques epistemológicos de las ciencias", "la comunicación de los saberes científicos", "reflexiones críticas y analíticas sobre los contenidos de los programas de las ciencias", "didáctica de las ciencias", "metodología de la investigación en didáctica de las ciencias"... La orientación de todos estos cursos demuestra que la problemática de la docencia universitaria está caracterizada desde la perspectiva de sus propios contenidos científicos, y enmarcada por su referencia a la misma ciencia. Ahora bien, la docencia universitaria deberá asimismo articular al eje ciencia - estudiante un eje transversal referido a la realidad social, cuya contextualización no puede dejar de hacerse presente en el proceso de la formación universitaria. 